\title{
Development and evaluation of an open source Delphi-based software for morphometric quantification of liver fibrosis
}

\author{
Sebastian Huss*1, Jörg Schmitz' ${ }^{1}$ Diane Goltz ${ }^{1}$, Hans-Peter Fischer ${ }^{1}$, Reinhard Büttner ${ }^{1}$ and Ralf Weiskirchen ${ }^{2}$
}

\begin{abstract}
Background: Computer-based morphometry can minimize subjectivity in the assessment of liver fibrosis. An image processing program was developed with Delphi for the quantification of fibrosis in liver tissue samples stained with Sirius Red. Bile duct ligated and sham operated wild type C57BL/6 mice served as a model of time-dependent induction of liver fibrosis. Formation of fibrosis was determined with the developed software at day $0,3,7,10,14,20,30$ and 60. The results were compared to a semi-quantitative scoring system.

Results: Quantitative accumulation of collagen fibres was observed from day 3 to day 14 , with a slight further increase thereafter. During ongoing fibrogenesis, there was a significant elevation of alanine aminotransferase (ALT), aspartate transaminase (AST) and bilirubin. The results from our computer-based morphometric analysis were highly correlated with the results that were obtained in a standardized pathology semi-quantitative scoring system $\left(R^{2}=0.89, n=38\right)$.

Conclusions: Using our Delphi-based image analysing software, the morphometric assessment of fibrosis is as precise as semi-quantitative scoring by an experienced pathologist. This program can be a valuable tool in any kind of experimental or clinical setting for standardized quantitative assessment of fibrosis.
\end{abstract}

\section{Background}

Liver fibrosis is characterized by an increase of collagenous matrix (the quantitative aspect of fibrosis). There is also a reduction of the vascular bed, pathologic perfusion, atrophy and regeneration of parenchyma leading to a fundamental rebuilding of tissue architecture (qualitative aspect of fibrosis). Complete organ fibrosis represents the final course of chronic progressive liver diseases.

Pathologists describe changes in these two dimensions subjectively or semi-quantitatively by a variety of scoring systems depending on the underlying disease causing different histological patterns. For example, in chronic hepatitis the Ishak score [1] is used. In alcoholic or non alcoholic steatohepatitis, fibrotic progression has been quantified by Brunt and colleagues [2]. Cholangiodestructive and cholangitic diseases are scored according to Portmann and Nakanuma [3]. A computer-based morphometry for the assessment of liver fibrosis is currently

\footnotetext{
*Correspondence: sebastian.huss@ukb.uni-bonn.de

1 Institute of Pathology, University of Bonn, Bonn, Germany Full list of author information is available at the end of the article
}

not in use for standard clinical diagnostics but is sometimes used scientifically in experimental models [4-8].

The primary goal of the present study was the development of highly user-friendly, charge-free and open source computer software to assess the quantitative aspect of liver fibrosis in a standardized and reproducible manner. Therefore, we used the model of bile duct ligation (BDL) to induct liver fibrosis in C57/BL6 mice. The experimental model has been well described and evaluated in rats and mice $[9,10]$ and has been widely used to study cholestatic liver injury $[11,12]$ and fibrogenesis $[9,13,14]$. We analysed time-related quantitative and semi-quantitative aspects of murine liver fibrosis and evaluated the different measurement techniques.

\section{Results}

Impact of bile duct ligation on survival, activity and jaundice

We have performed BDL in 40 animals and analysed ongoing hepatic fibrogenesis in respective animals at fixed time points (Figure 1). All sham-operated animals survived but two of 40 mice in the BDL group became 


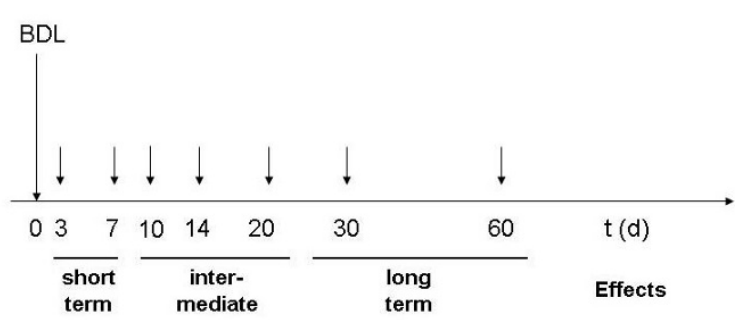

Figure 1 Study design and experimental setting. Bile duct ligation was performed on day 0 . Analysis was performed on day 3 and 7 after the procedure to measure short time effects. Time-points after 10, 14 and 20 days were chosen to display intermediate and 30 and 60 days to analyse long-term effects.

moribund and were killed before the planned end point. The activity of sham-operated animals was nearly normal $24 \mathrm{~h}$ after the lapratomy while the animals subjected to BDL showed reduced activity during the first $72 \mathrm{~h}$ but regained normal activity thereafter. Jaundiced skin was already apparent in all animals $24 \mathrm{~h}$ after BDL.

\section{Hepatocellular injury and cholestasis after BDL}

Alanine aminotransferase (ALT) and aspartate transaminase (AST) increased rapidly after BDL, peaking at 7 respectively twenty days after the surgery. After the peaking, ALT and AST decreased steadily until day 30; serum levels remained almost unchanged after 60 days. Bilirubin levels steadily elevated and reached a plateau after 7 days. Total protein serum levels had a greater variability with a slight decrease after 7 days (Figure 2).

\section{Development of liver fibrosis}

The stage of fibrosis was assessed semi-quantitatively. Periportal fibrosis was staged 0-4 and perisinusoidal fibrosis was scored $0-2$ by an experienced pathologist (HPF), giving a maximum possible of 6 (Table 1). The morphometric computer-based assessment of fibrosis showed $0.13 \pm 0.037 \%$ fibrosis-index in the sham operated group. A strong accumulation of collagen fibres was observed between day $3(0.10 \pm 0.03 \%)$ and $14(4.75 \pm$ $0.35 \%$ ), with a slight further increase thereafter (Table 2, Figure 3). The mean fibrosis score in sham operated animals was $0.00 \pm 0$. It increased steadily until day 60 to $4.83 \pm 0.17$. The maximum of periportal fibrosis (stage 3 ; complete lamellae) was reached at day $20(3.0 \pm 0.0)$. Peripsinusoidal fibrosis was absent during the first 10 days and was established after 14 days $(1.0 \pm 0.0)$. It increased steadily until day 60 to $1.8 \pm 0.17$ (Table 2, Figure 3).

Both the total and periportal semi-quantitative scoring values showed a good correlation with the computerbased assessment and could be fitted to a linear regres- sion curve ( $\mathrm{R} 2=0.89$ versus $\mathrm{R} 2=0.86 ; \mathrm{n}=38$ ). However, semi-quantitative scoring of perisinusoidal fibrosis showed a lower correlation $(\mathrm{R} 2=0.64, \mathrm{n}=38)$ (Figure 4).

\section{Discussion}

Liver fibrosis is a major parameter guiding the diagnosis and prognosis of chronic liver diseases and liver biopsy and its histological evaluation remains the gold standard for diagnosis and prognosis. Therefore, accurate qualitative and quantitative assessment of fibrosis is essential. Many scoring systems were designed to classify and stage different chronic liver diseases $[1,2,15]$. One major flaw of these scoring systems is that they are dependent on the visual interpretation of the observer. In addition, the observer must be an experienced pathologist. In order to avoid these pitfalls, over the last decade quantification of fibrosis by multiple computer assisted methods has been introduced $[5,7,16,17]$. The principle behind these methods is to stain a section with a specific tissue stain that highlights collagen fibres. Then, with the aid of the software, the fibrotic area is calculated. Ideally, the correct assessment of total fibrosis should be possible fully computerized that rules out intra- and inter-observer variations. Dahab et al. used the commercial program Adobe Photoshop to calculate a fibrosis index [6]. In addition, there are different companies (Soft Imaging Systems, Münster, Germany or Aperio, CA, USA) who sell programs which have been designed specially for image analysis purpose. These programs are usually highly expensive and are not easily adjustable.

Therefore, we have written a new simple, charge free, computer-based morphometric assessment tool to evaluate liver fibrosis. This software (Fibromat) is written in Delphi - one of the best known and widely used programming tools - to create software and applications for Microsoft Windows computers. With Delphi it is possible to create small powerful applications which do not need to be installed, do not depend on Active $\mathrm{X}$ controls or special DLLS. Therefore, there are no problems with installation, as usually observed with software from other programming utilities (for example, Java, .NET, Visual Basic or Visual $\mathrm{C}++_{+}$. The program allows the determination of different colour intensities that are necessary when morphometric scoring is necessary, for example, Sirus Red stained specimen. In order to perform a quantitative analysis, respective images of such a stain are first transformed into a grey scale and then further modified into a colour image in which the calculated background is depicted in black, liver parenchyma in light green and fibrous deposits in red. After this conversion, a precise and rapid mathematical quantification (in \%) of individual colours is possible. 


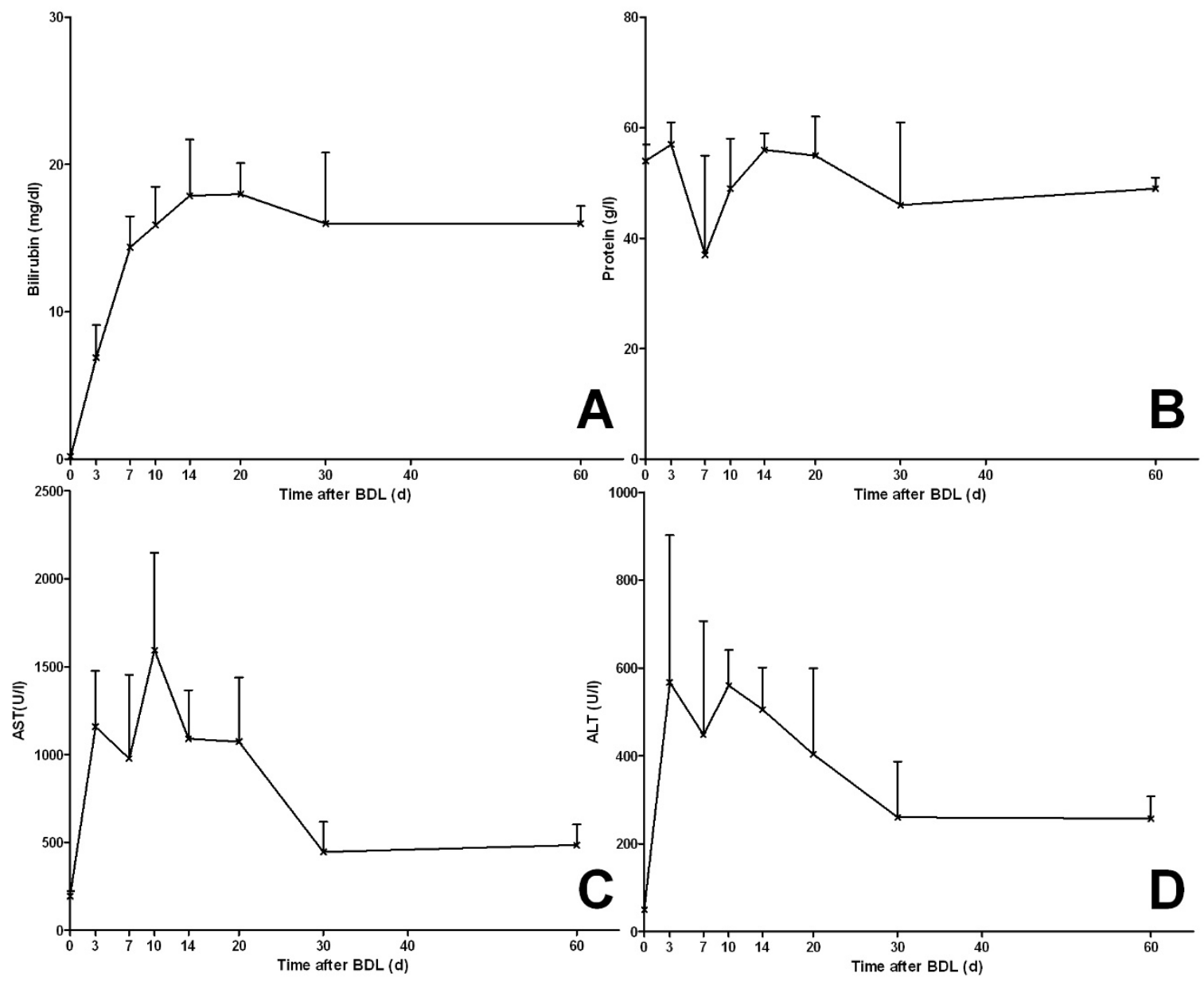

Figure 2 Serum markers of cholestasis and hepatocellular injury during bile duct ligation. Biochemical markers are shown for (A) bilirubin, (B) total protein, (C) alanine aminotransferase and (D) aspartate transaminase.

One major advantage of our program is that it is open source. Therefore, the complete source code of the program is accessibly by downloading it ( $\mathbf{T}$ Additional file $\mathbf{1}$ ) and it is easily adapted it to specific needs. For example one can choose different colors for the morphometrical analysis and make the program suitable for Massons Tricrome Staining or any immunohistochemical staining. With little effort an analysis of different subtypes of collagen is possible.

As previously mentioned, the pattern of fibrosis depends on the aetiology, severity and duration of the underlying disease. We investigated the qualitative and quantitative aspects of murine liver fibrosis caused by bile duct ligation using a semi-quantitative score. The pattern of fibrosis caused by this procedure can be compared, to a certain degree, to the pattern occurring in primary biliary cirrhosis ( $\mathrm{PBC})$. There is a type of periportal fibrosis that, along with the proliferation of neoductules and periductular fibrosis, leads to porto-portal septae [18]. A semi-quantitative scoring system for $\mathrm{PBC}$ was first described in 1965 by Rubin et al. [19]. They described four successive stages, which were modified by others [20-22] and summarized by Portmann and Nakanuma [3]. Other work groups scored their BDL-experiments according to one of these systems [23,24].

However, the scoring systems discussed were originally designed to score $\mathrm{PBC}$, an autoimmune liver disease with an unknown aetiology where selectively small intrahepatic bile ducts are destroyed. The pathogenic mechanism has to be distinguished from that occurring in BDL, where the common bile duct is ligated. Secondary to this procedure, small bile ducts react and form neoductuli [9].

We propose an alternative approach to assess fibrotic changes after murine bile duct ligation with a two-tired 
Table 1: Semi-quantitative scoring of fibrosis: a two-tired scoring system for histopathological liver changes after murine bile duct ligation

\begin{tabular}{lc}
\hline Periportal fibrosis & Score \\
\hline No fibrosis & 0 \\
\hline $\begin{array}{l}\text { Scattered periportal and perineoductular fibrosis } \\
\text { (incomplete lamellae) }\end{array}$ & 1 \\
\hline $\begin{array}{l}\text { Periportal, perineoductular fibrosis (complete lamellae) +/- } \\
\text { beginning septa }\end{array}$ & 2 \\
\hline \begin{tabular}{l} 
Periportal, perineoductular fibrosis with portal-portal septa * \\
\hline
\end{tabular} & 3 \\
\hline Complete cirrhosis & 4 \\
\hline
\end{tabular}

\section{Perisinusoidal fibrosis}

\begin{tabular}{lr}
\hline No fibrosis & 0 \\
\hline \begin{tabular}{l} 
Mild fibrosis (fibres in $<50 \%$ of the perisinusoidal spaces) \\
\hline
\end{tabular} & 1 \\
\hline Severe fibrosis (fibres in $>50 \%$ of the perisinusoidal spaces) & 2 \\
\hline *Three or more portal-portal septa have to be found per 10 portal \\
fields.
\end{tabular}

scoring system. Modified after Portmann and Nakanuma [3] we staged periportal fibrosis as follows: 0 , no fibrosis; stage 1 , focal periportal and perineoductular fibrosis that build incomplete lamellae around affected portal fields; stage 2, fully established periportal and perineoductular fibrosis building complete lamellae, with or without sporadic portal-portal bridging; stage 3 , an extension of the portal-portal bridging (three or more bridges per 10 portal fields); and stage 4, fully developed cirrhosis (Additional File 2). However, stage 4 was not observed in our experimental setting. This finding is different to descriptions of the rat model. Here fibrosis is progressive and cirrhosis can develop within 15 days after BDL [25]. Furthermore, we staged perisinusoidal fibrosis as follows: 0 , no fibrosis; stage 1, a mild fibrosis with less than $50 \%$ of the lobule affected; and stage 2, a severe fibrosis with more than $50 \%$ of the lobule affected.

In our model, periportal fibrosis was fully developed after 20 days; persinusoidal fibrosis become evident at day 10 and increased until the end of the experiment (day 60). When semi-quantitative scoring data are evaluated, it is important to bear in mind that the numbers represent categories rather than measurements. They, therefore, cannot be used as real numbers in statistical analyses [26,27]. Also adding the numbers representing different grading components (periportal and perisinusoidal) together to create a total score can lead to inaccuracies. At best, a total grading score can give only an approximate idea of the severity of disease [28]. We, therefore, correlated every dimension as well as the total score of our two-tired scoring system with the calculated fibrotic-index. We could show that the periportal fibrosis, as well as total score, is highly correlated but perisinusoidal fibrosis is lower. These findings suggest that a qualitative assessment of the computerized fibrosis pattern might still be necessary for an accurate interpretation of computerized fibrosis ratio, because a merely quantitative fibrotic-index of a specimen does not display all the information supplied by a visual interpretation of the slide. Nevertheless both, the semi-quantitatively scoring and the computer-based assessment (fibrotic-index) showed good correlation in depicting increased collagen deposition as a consequence of ongoing fibrogenesis. Therefore, we propose this highly user-friendly image analysis tool for the accurate quantification of collagen deposits in Sirius Red stained liver sections. The image processing is computerized and, for this reason, more insensitive to intra- and inter-observer variations than a semi-quantitative scoring system.

\section{Conclusions}

We designed an easy-to-use, charge free and open source Delphi-based computer program to assess the quantitative aspect of liver fibrosis in a standardized and reproducible way. The program was evaluated in an experimental setting of murine liver fibrosis following bile duct ligation. It can also be used for the analysis of fibrosis due to other aetiopathologies.

\section{Methods \\ Animals}

Male C57BL/6 mice (Harlan Laboratories, Eystrup, Germany) aged 10-12 weeks were kept under controlled environmental conditions with a 12-h light-dark cycle for a minimum of 7 days before surgery. Mice were fed on a standard laboratory diet with food and water ad libitum. All experiments were approved by the Landesamt für Natur, Umwelt und Verbraucherschutz NRW, Recklinghausen, Germany (AZ 50.203.2 AC20, 13/06 and AZ 8.87-50.10.37.09.248).

\section{Procedures}

$B D L$

After midline skin lapratomy the liver was gently removed and the common bile duct was mobilized. In the region above the pancreas two 4-0 nylon sutures were 


\begin{tabular}{|c|c|c|c|c|}
\hline \multirow[t]{2}{*}{ Time after bile duct ligation (days) } & \multirow[t]{2}{*}{ Fibrosis index (\%) } & \multicolumn{3}{|c|}{ Semi-quantitative score } \\
\hline & & Portal fibrosis & Perisinusoidal fibrosis & Total score \\
\hline $0(n=3)$ & $0.13 \pm 0.04$ & $0.0 \pm 0.0$ & $0.0 \pm 0.0$ & $0.0 \pm 0.0$ \\
\hline $3(n=5)$ & $0.10 \pm 0.03$ & $0.0 \pm 0.0$ & $0.0 \pm 0.0$ & $0.0 \pm 0.0$ \\
\hline $7(n=5)$ & $0.76 \pm 0.11$ & $0.60 \pm 0.25$ & $0.0 \pm 0.0$ & $0.60 \pm 0.25$ \\
\hline $10(n=5)$ & $1.65 \pm 0.22$ & $1.40 \pm 0.25$ & $0.25 \pm 0.25$ & $1.67 \pm 0.25$ \\
\hline $14(n=5)$ & $4.75 \pm 0.35$ & $2.4 \pm 0.25$ & $1.0 \pm 0.0$ & $3.40 \pm 0.24$ \\
\hline $20(n=4)$ & $5.02 \pm 0.47$ & $3.0 \pm 0.0$ & $1.0 \pm 0.0$ & $4.0 \pm 0.0$ \\
\hline $30(n=5)$ & $5.45 \pm 0.35$ & $2.8 \pm 0.2$ & $1.4 \pm 0.25$ & $4.20 \pm 0.20$ \\
\hline $60(n=6)$ & $5.63 \pm 0.25$ & $3.0 \pm 0.0$ & $1.8 \pm 0.17$ & $4.83 \pm 0.17$ \\
\hline
\end{tabular}

Fibrosis index (\% Sirius Red stain) and semi-quantitative score consisting of portal fibrosis score, perisinusoidal score and total score (mean $+/-$ standard error of mean; see Table 1)

placed around the bile duct and carefully tightened to avoid rupture.

\section{Sham lapratomy (control)}

Lapratomy and mobilization of the common bile duct were performed as in the former group but without ligation.

In order to measure short time effects of the BDL, mice were sacrificed at day 3 and 7 after the surgery. In order to indicate intermediate time effects mice were sacrificed after 14 and 20 days, to assess long term effects after 30 and 60 days. Control animals were killed 20 days after the sham lapratomy. At indicated time points, blood was drawn from the right ventricle, centrifuged and the serum was stored at $-80^{\circ} \mathrm{C}$ until further analysis. The liver was removed and fixed in $4 \%$ buffered formalin for $24 \mathrm{~h}$ for histological analysis.

\section{Measurement of serum parameters}

Blood biochemical parameters (bilirubin, ALT, AST and total protein) were measured using the Modular PreAnalytics (MPA) system (Roche Diagnostics, Mannheim, Germany)

\section{Histology}

After being fixed in $4 \%$ buffered formalin for $24 \mathrm{~h}$, the liver tissue was embedded into paraffin wax. A histological semi-quantitative examination of the liver was performed on sections after standard Sirius Red staining.
Periportal fibrosis was staged $0-4$ and perisinusoidal fibrosis was scored 0-2, giving a maximum possible of 6 (Table 1).

\section{Development of the image analysing program}

The principle behind computer-based morphometry is the different staining pattern of cells, nuclei and fibres following Sirius Red staining. Collagen fibres, as well as cell nuclei, appear red, while the hepatocellular cytoplasm becomes pale and yellowish.

For the analysis, 10 photographs of random high-power fields $(100 \times$ magnifications) were taken of each liver sample. Large bile ducts and vessels were excluded. Photographs were stored as $1280 \times 1024$ pixel RGB-bitmaps (bmp) with a colour-resolution of 24 bits per pixel. These pictures were analysed with our Delphi-based program as follows. After a shading correction, a grey transformation - derived from the green color channel only - is calculated from the original image. Then a blurring filter based on an arithmetic mean filter is applied. Thresholding of the positively stained collagen fibres is performed by a histogram analysis of the grey value distribution resulting in a binary image. As cell nuclei and fibres appear to have the same staining intensity, binary object detection is performed and all objects with an area lower than a specific threshold are eliminated, distinguishing between fibres and nuclei. The total area of the combined fibres is expressed as a percentage of the total parenchyma area. 


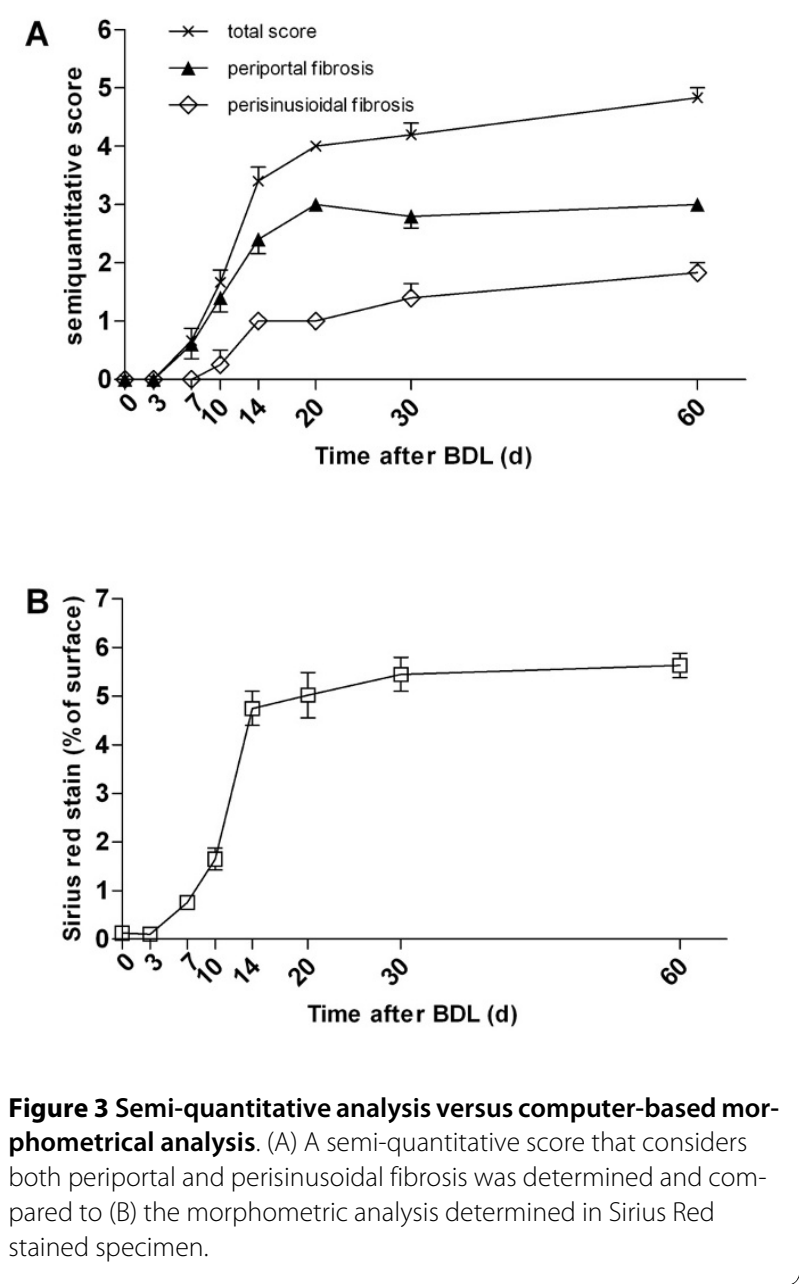

These steps are performed automatically and the displayed resulting image shows background in black, liver parenchyma in a light green and fibrosis intense red (Figures 5 and 6). Nevertheless, the user has several options to interact with the program, eliminate different common errors and to simplify his work. (i) Sirius Red staining can have different intensities due to staining time and/or thickness of the histological slide. The user can modify the threshold of the grey transformation to minimize these effects. (ii) If random sections of a sample were taken there may be flapped areas or other artifacts giving a wrong signal. The user can exclude obviously mistaken objects by a simple mouse click. All steps of the complete procedure are visualized in one display and are therefore easy to follow. (iii) Lumina of small vessels, as well as the background, are automatically excluded from the total area of the parenchyma. (iv) Unlimited photographs can be analysed one after another. The user can save and reopen the current working file or send it to a co-worker who can proceed or reevaluate the work. (v) The values
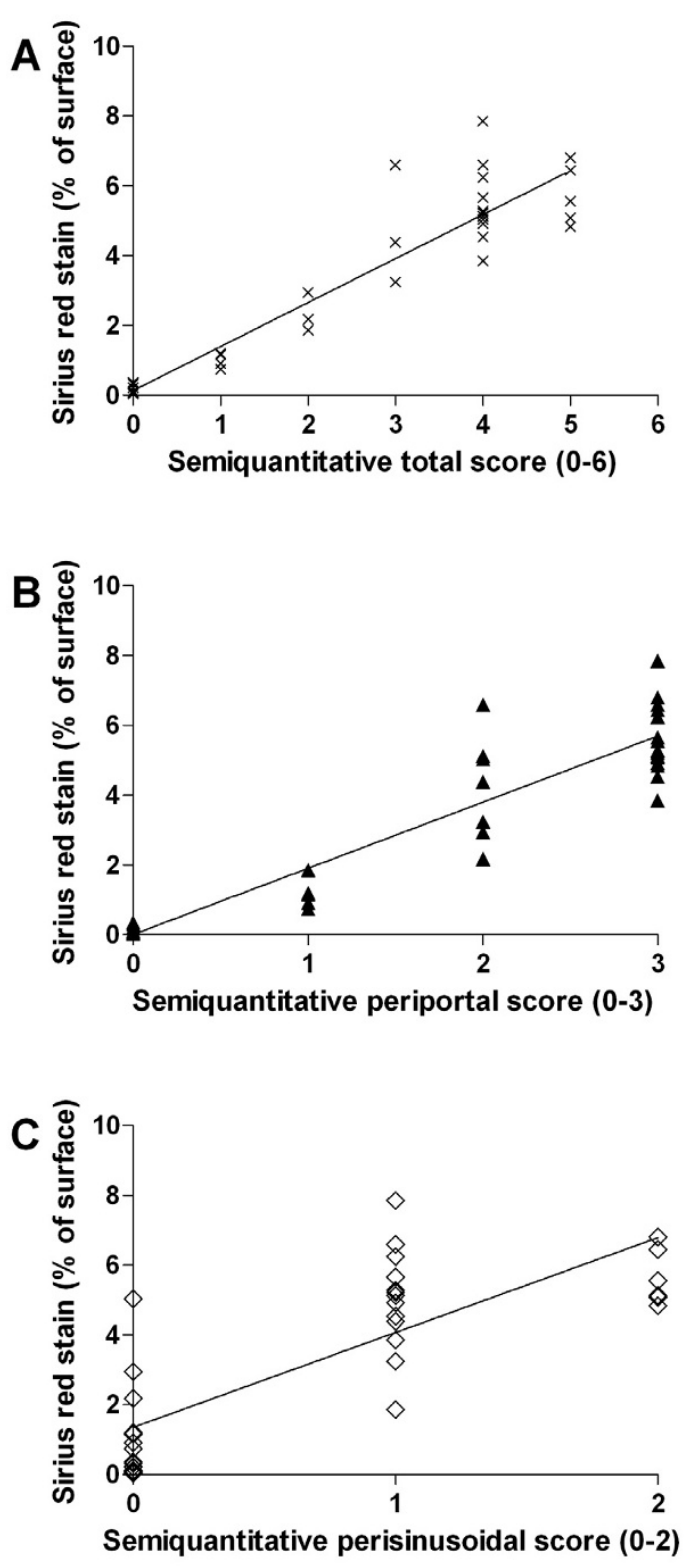

Figure $\mathbf{4}$ Correlation between semi-quantitative and computerbased analysis. Linear regression fit between semi-quantitative total $(\mathrm{A} ; R 2=0.89)$, periportal $(\mathrm{B} ; R 2=0.86)$ and perisinuidal $(C ; R 2=0.64)$ score and morphometric assessment (Percentage of positively stained surface, Sirius Red Staining; $n=38$.)

(0.00-1.00, that is $0 \%$ to $100 \%$ fibrosis) can be exported to a text file and re-imported into Excel, GraphPad, SPSS or another program for statistical analysis. The software, which is compatible with Microsoft Windows 7, Microsoft Vista SP2, Microsoft Windows XP Home or Professional (SP2 or SP3), can be downloaded from the journal home page (Additional File 1). Details of programming are available on request. 

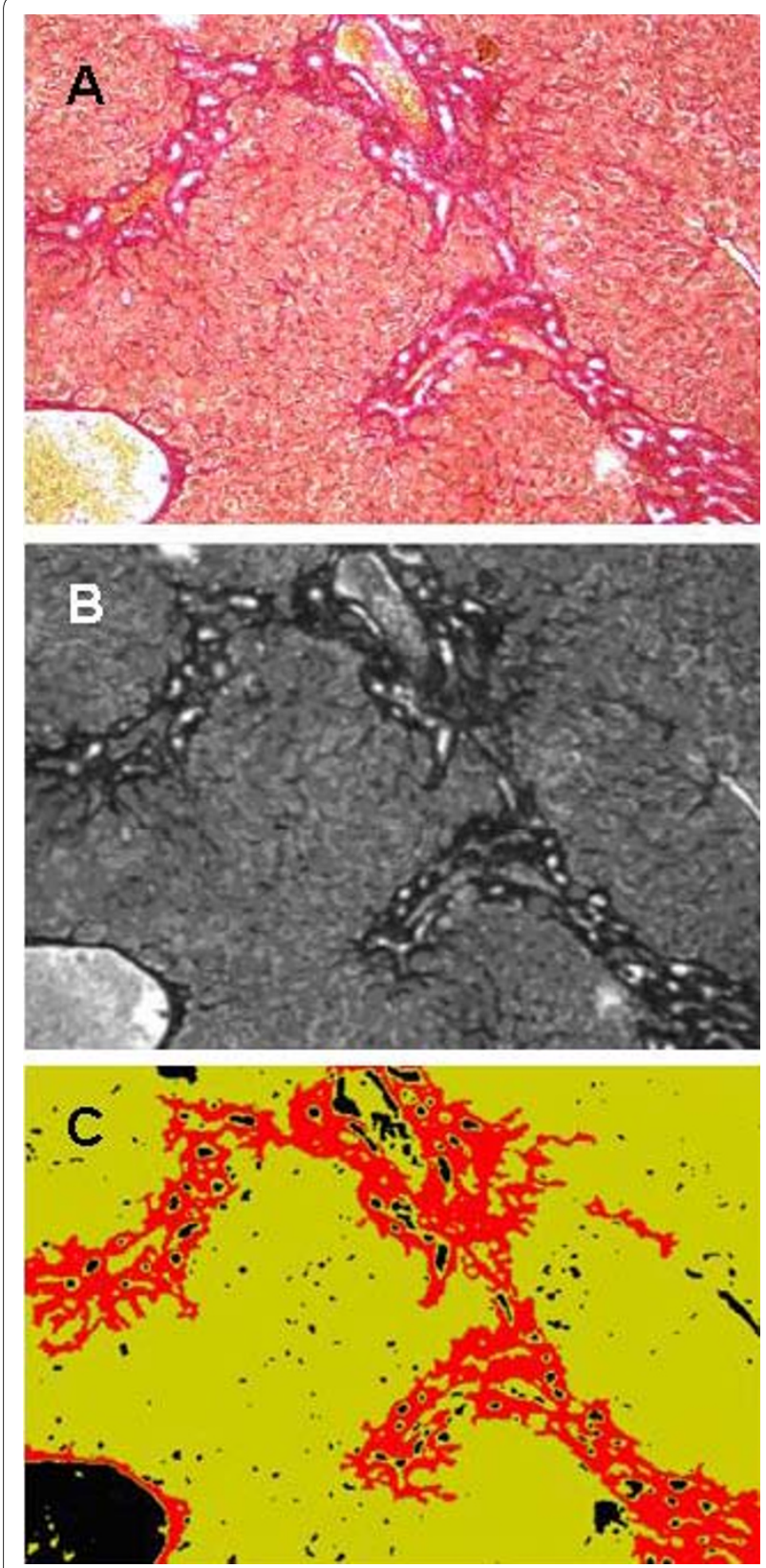

Figure 5 Computer-based morphometry. (A) Photograph of a Sirius Red stain of a liver section (100 $\times$ magnifications). (B) Grey transformation, blurring and shading of respective photograph. (C) Resulting image of respective photograph. Background is black, liver parenchyma light green and fibrosis intuitively red.

\section{Statistical analysis}

All results are expressed as the mean values \pm standard error of mean, except for serum parameters of the animals, which are expressed as mean values \pm standard deviations. Semi-quantitative scores were correlated with the values from the computer assisted morphometrical analysis using Graph Pad Prism software.

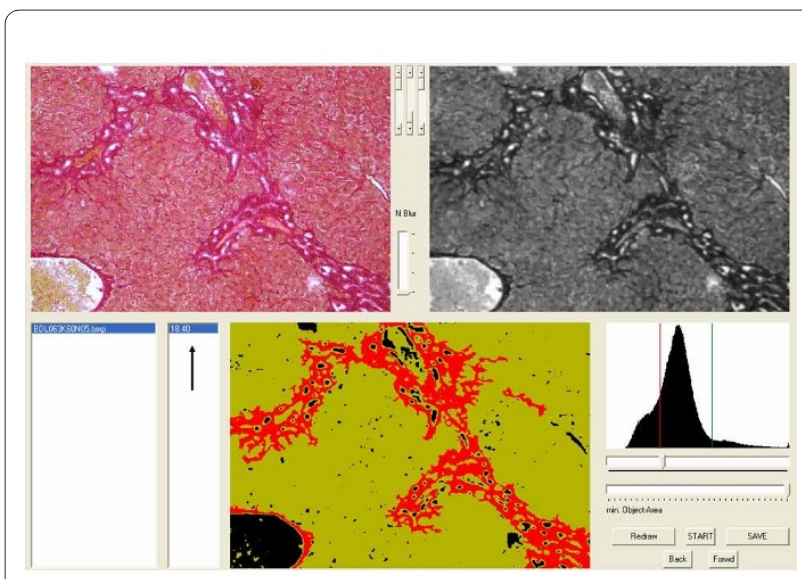

Figure $\mathbf{6}$ Screenshot of program interface. The original photographs are shown in the upper left corner. In the upper right corner the same picture is shown after adding the grey transformation, blurring and shading. The histogram analysis is shown in the lower right corner. The image in the lower middle shows the calculated background in black, the liver parenchyma in a light green and fibrosis intuitively red. The percentage of fibrous tissue is calculated on the lower left side (black arrow).

\section{Additional material}

Additional file 1 The Fibromat, an open source Delphi-based software for computer-based morphometry. Download the file and unzip it to your hard disc. Open Delphi (installation is required; program can be obtained from Borland, Texas, USA). Open the file 'ihc.exe' and start the program. If you have any questions on how to use or modify the program feel free to contact us via the corresponding author.

Additional file $\mathbf{2}$ Scoring periportal fibrosis. (A) A normal portal field without fibrosis (stage 0). (B) Focal periportal and perineoductular fibrosis (incomplete lamellae, stage 1). (C) Fully established periportal and perineoductular fibrosis building complete lamellae, with or without sporadic portal-portal bridging (stage 2). (D) Extension of the portal-portal bridging (three or more bridges per 10 portal fields, stage 3); complete cirrhosis (stage 4) is not shown.

\section{Abbreviations}

ALT: alanine aminotransferase; AST: aspartate transaminase; BDL: bile duct ligation; bmp: bitmaps; PBC: primary biliary cirrhosis.

\section{Competing interests}

The authors declare that they have no competing interests.

\section{Authors' contributions}

SH did the animal experiments, helped with the development of the program and drafted the manuscript. JS was the principle programmer and performed the quantitative analysis. DG helped with the animal experiments. RB supervised the animal experiments and participated in the study design. HPF performed the histopathological semi-quantitative scoring. RW was responsible for the serum analysis and was involved in the study design as well as in revising the manuscript for important intellectual content. All authors carefully read and approved the final manuscript.

\section{Acknowledgements}

The study was supported by SFB/TRR57 (projects P05 and Q1) from the Deutsche Forschungsgemeinschaft (DFG). The authors thank Christiane Esch and Claudine Neumann for their excellent technical support. 


\section{Author Details}

'Institute of Pathology, University of Bonn, Bonn, Germany and 2Institute of Clinical Chemistry and Pathobiochemistry, RWTH University Hospital Aachen, Germany

Received: 22 April 2010 Accepted: 17 June 2010

Published: 17 June 2010

\section{References}

1. Ishak K, Baptista A, Bianchi L, Callea F, De Groote J, Gudat F, Denk H, Desmet V, Korb G, MacSween RN, et al:: Histological grading and staging of chronic hepatitis. J Hepatol 1995, 22:696-699.

2. Brunt EM: Nonalcoholic steatohepatitis: definition and pathology. Semin Liver Dis 2001, 21:3-16.

3. Diseases of the Bile Ducts. 5th edition. Edited by: Portmann BC, Nakanuma Y. New York: Churchill Livingstone; 2007.

4. Zaitoun AM, Mardini H Al, Awad S, Ukabam S, Makadisi S, Record CO: Quantitative assessment of fibrosis and steatosis in liver biopsies from patients with chronic hepatitis C. J Clin Pathol 2001, 54:461-465.

5. Caballero T, Perez-Milena A, Masseroli M, O'Valle F, Salmeron FJ, Del Moral RM, Sanchez-Salgado G: Liver fibrosis assessment with semiquantitative indexes and image analysis quantification in sustained-responder and non-responder interferon-treated patients with chronic hepatitis $C$. J Hepatol 2001, 34:740-747.

6. Dahab GM, Kheriza MM, El-Beltagi HM, Fouda AM, El-Din OA: Digital quantification of fibrosis in liver biopsy sections: description of a new method by Photoshop software. J Gastroenterol Hepatol 2004, 19:78-85.

7. Masseroli M, Caballero T, O'Valle F, Del Moral RM, Perez-Milena A, Del Moral RG: Automatic quantification of liver fibrosis: design and validation of a new image analysis method: comparison with semiquantitative indexes of fibrosis. J Hepatol 2000, 32:453-464.

8. Abdalla AF, Zalata KR, Ismail AF, Shiha G, Attiya M, Abo-Alyazeed A: Regression of fibrosis in paediatric autoimmune hepatitis: morphometric assessment of fibrosis versus semiquantiatative methods. Fibrogenesis Tissue Repair 2009, 2:2.

9. Georgiev P, Jochum W, Heinrich S, Jang JH, Nocito A, Dahm F, Clavien PA: Characterization of time-related changes after experimental bile duct ligation. Br J Surg 2008, 95:646-656.

10. Johnstone JM, Lee EG: A quantitative assessment of the structural changes the rat's liver following obstruction of the common bile duct. Br J Exp Pathol 1976, 57:85-94.

11. Wang H, Vohra BP, Zhang Y, Heuckeroth RO: Transcriptional profiling after bile duct ligation identifies PAI-1 as a contributor to cholestatic injury in mice. Hepatology 2005, 42:1099-1108.

12. Fickert P, Zollner G, Fuchsbichler A, Stumptner C, Weiglein AH, Lammert F, Marschall HU, Tsybrovskyy O, Zatloukal K, Denk H, Trauner M: Ursodeoxycholic acid aggravates bile infarcts in bile duct-ligated and Mdr2 knockout mice via disruption of cholangioles. Gastroenterology 2002, 123:1238-1251.

13. Neumeier M, Hellerbrand C, Gabele E, Buettner R, Bollheimer C, Weigert J, Schaffler A, Weiss TS, Lichtenauer M, Scholmerich J, Buechler C: Adiponectin and its receptors in rodent models of fatty liver disease and liver cirrhosis. World J Gastroenterol 2006, 12:5490-5494.

14. Arias M, Sauer-Lehnen S, Treptau J, Janoschek N, Theuerkauf I, Buettner R, Gressner AM, Weiskirchen R: Adenoviral expression of a transforming growth factor-beta1 antisense mRNA is effective in preventing liver fibrosis in bile-duct ligated rats. BMC Gastroenterol 2003, 3:29.

15. Ludwig J, Dickson ER, McDonald GS: Staging of chronic nonsuppurative destructive cholangitis (syndrome of primary biliary cirrhosis). Virchows Arch A Pathol Anat Histol 1978, 379:103-112.

16. Nohlgard $C$, Rubio $C A$, Kock $Y$, Hammar H: Liver fibrosis quantified by image analysis in methotrexate-treated patients with psoriasis. JAm Acad Dermatol 1993, 28:40-45.

17. O'Brien MJ, Keating NM, Elderiny S, Cerda S, Keaveny AP, Afdhal NH, Nunes DP: An assessment of digital image analysis to measure fibrosis in liver biopsy specimens of patients with chronic hepatitis C. Am J Clin Pathol 2000, 114:712-718.

18. Beaussier M, Wendum D, Schiffer E, Dumont S, Rey C, Lienhart A, Housset C: Prominent contribution of portal mesenchymal cells to liver fibrosis in ischemic and obstructive cholestatic injuries. Lab Invest 2007 87:292-303
19. Rubin E, Schaffner F, Popper H: Primary Biliary Cirrhosis. Chronic NonSuppurative Destructive Cholangitis. Am J Pathol 1965, 46:387-407.

20. Kaplan MM, Gershwin ME: Primary biliary cirrhosis. N Eng/ J Med 2005, 353:1261-1273

21. Lucey MR, Neuberger JM, Williams R: Primary biliary cirrhosis in men. Gut 1986, 27:1373-1376.

22. Scheuer P: Primary biliary cirrhosis. Proc R Soc Med 1967, 60:1257-1260

23. Jonsson JR, Clouston AD, Ando Y, Kelemen LI, Horn MJ, Adamson MD, Purdie DM, Powell EE: Angiotensin-converting enzyme inhibition attenuates the progression of rat hepatic fibrosis. Gastroenterology 2001, 121:148-155.

24. Park DH, Baik SK, Choi YH, Kim MY, Rhim DW, Kim JW, Kwon SO, Cho MY, Kim CH, Ahn SC: Inhibitory effect of angiotensin blockade on hepatic fibrosis in common bile duct-ligated rats. Korean J Hepatol 2007 13:61-69.

25. Kountouras J, Billing BH, Scheuer PJ: Prolonged bile duct obstruction: a new experimental model for cirrhosis in the rat. Br J Exp Pathol 1984 65:305-311.

26. Cross SS: Grading and scoring in histopathology. Histopathology 1998 33:99-106.

27. Macnaughton RJ: Numbers, scales and qualitative research. Lancet 1996, 347:1099-1100.

28. Theise ND BH, Ferrell LD, (Ed): Acute and chronical viral hepatitis. 5th edition. New York: Churchill Livingstone; 2007.

doi: $10.1186 / 1755-1536-3-10$

Cite this article as: Huss et al., Development and evaluation of an open source Delphi-based software for morphometric quantification of liver fibrosis Fibrogenesis \& Tissue Repair 2010, 3:10

\section{Submit your next manuscript to BioMed Central and take full advantage of:}

- Convenient online submission

- Thorough peer review

- No space constraints or color figure charges

- Immediate publication on acceptance

- Inclusion in PubMed, CAS, Scopus and Google Scholar

- Research which is freely available for redistribution
C Biomed Central 